\title{
Bayesian Value-at-Risk and Expected Shortfall for a Large Portfolio (Multi- and Univariate Approaches)
}

\author{
A. PAJOR AND J. OSIEWALSKI \\ Department of Econometrics and Operations Research, Cracow University of Economics \\ Rakowicka 27, 31-510 Kraków, Poland
}

\begin{abstract}
Bayesian assessments of value-at-risk and expected shortfall for a given portfolio of dimension $n$ can be based either on the $n$-variate predictive distribution of future returns of individual assets, or on the univariate model for portfolio volatility. In both cases, the Bayesian VaR and ES fully take into account parameter uncertainty and non-linear relationship between ordinary and logarithmic returns. We use the $n$-variate type I MSF-SBEKK $(1,1)$ volatility model proposed specially to cope with large $\mathrm{n}$. We compare empirical results obtained using this (more demanding) multivariate approach and the much simpler univariate approach based on modelling volatility of the whole portfolio (of a given structure).
\end{abstract}

PACS: 89.65.Gh, 87.55.kh, 02.50.Sk.

\section{Introduction}

For investors and banks accurate evaluation of risk is very important. An underestimation of risk could throw them into bankruptcy. On the other hand, an overestimation of risk may have a negative effect on their profits. Two risk measures are very popular: value-at-risk (VaR) and expected shortfall (ES).

The VaR measures the least portfolio loss that may happen with a given probability $\alpha$ (usually 0.05 or smaller) over a certain time horizon (most often from 1 to 10 trading days). From mathematical point of view the VaR is a quantile of the Profit-and-Loss distribution of a given portfolio over a certain time horizon. A disadvantage of this measure is that it does not inform us about the potential size of loss that exceeds the VaR. Moreover, the VaR is not a coherent measure - it lacks the property of sub-additivity, i.e. the VaR of a portfolio may be greater than the sum of the VaR levels of the constituents of the portfolio [1]. Despite theoretical discussions, the VaR has become the standard measure of market risk used both by financial institutions and by their regulators; see [2]. According to the Capital Adequacy Directive by the Bank of International Settlement in Basel, banks are required to hold a certain amount of capital as a protection against (among others) adverse movements in the market values of assets. A bank should possess sufficient capital "to cover losses on the bank's trading portfolio over a 10-day holding period in $99 \%$ of occasions"; see [3].

Expected Shortfall is defined as the conditional expectation of the loss given that it exceeds the VaR level; see [4]. Expected Shortfall is a sub-additive, coherent measure. The property of sub-additivity means that the ES for a portfolio is not greater than the sum of its subportfolios' ES.

The VaR and ES are characteristics of the distribution of the future portfolio value (conditional on historical data on asset prices) and are closely related to its tails. More popular and traditional approaches to the VaR and ES assessment are based on parametric sta- tistical models (with explicit assumptions on the conditional distribution of future returns) or methods related to the extreme value theory. Other approaches are based on non-parametric models, such as Conditional Autoregressive Expectiles (CARE, see [5]) and Conditional Autoregressive Value at Risk (CAViaR, see [2]). These approaches directly focus on the Expected Shortfall and on the $\alpha$-quantile modelled non-parametrically.

In this paper we discuss and compare VaR and ES assessments based on multi- and univariate parametric models. The multivariate approach is much more difficult, as it explicitly takes into account the full conditional covariance structure of asset prices: individual volatilities and correlations. Most of multivariate models in financial econometrics either belong to the Multivariate GARCH (MGARCH) or multivariate stochastic volatility (MSV) classes or are based on copulas; see [6,7]. These models are difficult to estimate; only a few of them could be practical tools for large portfolios. A solution to the problem of simple, but parsimonious, multivariate volatility modelling is a hybrid model proposed by Osiewalski [8], see also [9]. This hybrid model is based on scalar BEKK (SBEKK) correlation structure and the simplest MSV specification, the multiplicative stochastic factor (MSF) model.

The VaR and ES require only the distribution of the future value of the portfolio; it can be derived using a univariate model for the historical values of the portfolio.

In our comparison we use the Bayesian MSF-SBEKK type I model for portfolios of dimension $n=50$ and the univariate specification obtained from this model by taking $n=1$. In the next section we discuss basic notions and introduce notation. Section 3 is devoted to our models proposed for the assessment of VaR and ES. Section 4 contains empirical results. Section 5 concludes.

\section{Portfolio value-at-risk and expected shortfall - concepts and notation}

Consider a portfolio kept at present time $(T)$ and consisting of $n$ assets; $a_{i}$ denotes the number of units of 
asset $i$ possessed now and $S_{t, i}$ is the price of asset $i$ at time $t\left(S_{t, i}>0, a_{i}>0\right.$ for $i=1, \ldots, n, t=1, \ldots, T$ ) , thus $W_{t}=\sum_{i=1}^{n} a_{i} S_{t, i}$ is the time $t$ value of this portfolio. The $s$-period return rate on the portfolio is $R_{t: t+s}^{*}=\left(W_{t+s}-W_{t}\right) / W_{t}=\sum_{i=1}^{n} \omega_{t, i} R_{t: t+s, i}$, where $R_{t: t+s, i}=\left(S_{t+s, i}-S_{t, i}\right) / S_{t, i}$ is the $s$-period return rate on asset $i$ and $\omega_{t, i}=a_{i} S_{t, i} / W_{t}$ is the share of asset $i$ in the time $t$ portfolio value. For most results the condition $a_{i}>0$ is not required (short sale is allowed), only $W_{t}>0$ has to be assumed. Note that the sum of $\omega_{t, i}$ over the assets $(i=1, \ldots, n)$ is always 1 by construction.

Assume that we observe the $n$-variate time series of individual return rates for $t=1, \ldots, T$ and we are interested in forecasting $R_{T: T+s}^{*}$, the $s$-period ahead return on the portfolio kept at time $T$. Forecasting $R_{T: T+s}^{*}$ is closely related to the definition of $\operatorname{VaR}_{T: T+s}^{L}$ and $\operatorname{VaR}_{T: T+s}^{S}$, the $s$-period ahead Value-at-Risk (VaR) for, respectively, long and short trading positions of the portfolio. If $\Psi_{T}$ denotes the observations on asset prices up to time $T$, then $\operatorname{VaR}_{T: T+s}^{L}(\alpha)$ for a given probability level $\alpha$ is defined by the following equality:

$$
\operatorname{Pr}\left[W_{T+s} \leq W_{T}-\operatorname{VaR}_{T: T+s}^{L}(\alpha) \mid \Psi_{T}\right]=\alpha,
$$

$$
\operatorname{Pr}\left[R_{T: T+s}^{*} \leq \frac{-\mathrm{VaR}_{T: T+s}^{L}(\alpha)}{W_{T}} \mid \Psi_{T}\right]=\alpha .
$$

That is, the relative $s$-period ahead VaR for a long trading position (corresponding to some fixed, small $\alpha$ ) is the negative of the $\alpha$-quantile of the conditional distribution of the $s$-period ahead return on the portfolio, given $\Psi_{T}$, the information (on asset prices) available at time $T$. Similarly, we define the VaR for a short trading position:

$$
\operatorname{Pr}\left[W_{T+s} \geq W_{T}+\operatorname{VaR}_{T: T+s}^{S}(\alpha) \mid \Psi_{T}\right]=\alpha,
$$

which can be written as

$$
\operatorname{Pr}\left[R_{T: T+s}^{*} \geq \frac{\operatorname{VaR}_{T: T+s}^{S}(\alpha)}{W_{T}} \mid \Psi_{T}\right]=\alpha .
$$

The relative $s$-period ahead VaR for a short trading position is the $(1-\alpha)$-quantile of the conditional distribution of the $s$-period ahead return on the portfolio.

In equalities (2) and (4) we have the ordinary return rate $R_{T: T+s}^{*}$. In practice, the logarithmic return rates $r_{t+1, i}=\ln \left(S_{t+1, i} / S_{t, i}\right)=\ln \left(R_{t: t+1, i}+1\right)$ are the quantities being modelled. They can take any real value, easily aggregate over time, and modelling them can be more justified in view of the data; see [10]. Using the logarithmic return rates we can rewrite (2) and (4) as:

which can be written as

$$
\begin{aligned}
& \operatorname{Pr}\left[-1+\sum_{i=1}^{n} \omega_{T, i} \exp \left(\sum_{j=1}^{s} r_{T+j, i}\right) \leq \frac{-\operatorname{VaR}_{T: T+s}^{L}(\alpha)}{W_{T}} \mid \Psi_{T}\right]=\alpha, \\
& \operatorname{Pr}\left[-1+\sum_{i=1}^{n} \omega_{T, i} \exp \left(\sum_{j=1}^{s} r_{T+j, i}\right) \geq \frac{\operatorname{VaR}_{T: T+s}^{S}(\alpha)}{W_{T}} \mid \Psi_{T}\right]=\alpha,
\end{aligned}
$$

for details see [11].

The $s$-period ahead Expected Shortfall (ES) for a long trading position at a given probability level $\alpha$, denoted by $\operatorname{ES}_{T: T+s}^{L}(\alpha)$, is defined by

$$
\operatorname{ES}_{T: T+s}^{L}(\alpha)=-E\left[W_{T+s}-W_{T} \mid W_{T+s} \leq W_{T}-\operatorname{VaR}_{T: T+s}^{L}(\alpha), \Psi_{T}\right]
$$

and for a short trading position:

$$
\operatorname{ES}_{T: T+s}^{S}(\alpha)=E\left[W_{T+s}-W_{T} \mid W_{T+s} \geq W_{T}+\operatorname{VaR}_{T: T+s}^{S}(\alpha), \Psi_{T}\right] .
$$

Using the logarithmic return rates we can rewrite (7) and (8) as:

$$
\begin{aligned}
& \operatorname{ES}_{T: T+s}^{L}(\alpha)=-E\left[W_{T} \sum_{i=1}^{n} \omega_{T, i} \exp \left(\sum_{j=1}^{s} r_{T+j, i}\right)-W_{T} \mid W_{T+s} \leq W_{T}-\operatorname{VaR}_{T: T+s}^{L}(\alpha), \Psi_{T}\right], \\
& \operatorname{ES}_{T: T+s}^{S}(\alpha)=E\left[W_{T} \sum_{i=1}^{n} \omega_{T, i} \exp \left(\sum_{j=1}^{s} r_{T+j, i}\right)-W_{T} \mid W_{T+s} \geq W_{T}+\operatorname{VaR}_{T: T+s}^{S}(\alpha), \Psi_{T}\right],
\end{aligned}
$$

The VaR and ES are related to quantiles of some nonlinear function of future logarithmic returns*. Condi-

* The usual linear approximation $\exp \left(\sum_{j=1}^{s} r_{t+j, i}\right) \approx 1+\sum_{j=1}^{s} r_{t+j, i}$ tioning on observed data and inference on non-linear functions of unobserved quantities are natural within the Bayesian approach to statistics; see [12]. Therefore this

can lead to serious errors, especially when $s$ is so large that the $s$-period ahead return distribution is diffuse, see [11]. 
approach is advocated for determining the $s$-period ahead VaR and ES. Foundations of Bayesian VaR assessment are presented in [11]. The same approach is used here for the ES assessment. Similarly as in [11], we consider two modelling strategies for the portfolio VaR and ES. The first one amounts to assuming some $n$-variate model for individual logarithmic returns $r_{t, i}$ and obtaining the $\alpha$ - and (1- $\alpha)$-quantile of the predictive distribution of $R_{T: T+s}^{*}=-1+\sum_{i=1}^{n} \omega_{T, i} \exp \left(\sum_{j=1}^{s} r_{T+j, i}\right)$, a nonlinear function of future returns. The second approach amounts to directly modelling univariate series of portfolio logarithmic returns $r_{t+1}^{W}=\ln \left(W_{t+1} / W_{t}\right)$ and examining the predictive distribution of $r_{T: T+s}^{W}=\ln \left(W_{T+s} / W_{T}\right)$. The two approaches ( $n$ - and univariate) are not formally coherent and their comparison is an empirical task, undertaken in this paper. The question is whether a univariate specification from a flexible parametric family can explain and predict portfolio returns better than $n$-variate models that require huge simplifications in order to cope with large $n$. In the case of VaR assessments for a long trading position, the results presented by Osiewalski and Pajor [11] are not conclusive, although reasonable performance of our n-variate hybrid model is somewhat surprising. In this paper we also consider VaR assessments for a short trading position and the ES.

\section{The hybrid VAR(1)-MSF-SBEKK type I Bayesian model}

First we consider a multivariate specification for individual assets. Let $\mathbf{r}_{t}=\left[r_{t, 1} \ldots r_{t, n}\right]$ denote $n$-variate observations on logarithmic return rates, which we model using the basic $\operatorname{VAR}(1)$ framework:

$$
\mathbf{r}_{t}=\mathbf{d}_{0}+\mathbf{r}_{t-1} \boldsymbol{\Phi}+\mathbf{e}_{t}, \quad t=1, \ldots, T+s .
$$

The $n(n+1)$ elements of the row vector $\mathbf{d}=\left[\mathbf{d}_{0}(\operatorname{vec} \mathbf{\Phi})^{\prime}\right]^{\prime}$ are common parameters, which can be treated as a priori independent of all other (model-specific) parameters; we can assume for them some multivariate prior, e.g. standard Normal $N\left(\mathbf{0}, \mathbf{I}_{n(n+1)}\right)$, truncated by the restriction that all eigenvalues of $\boldsymbol{\Phi}$ lie inside the unit circle.

Following [9], we specify the conditional distribution of the residual process $\mathbf{e}_{t}$ by conditioning on its past $\left(\Psi_{t-1}\right)$, some univariate latent process $\left(g_{t}\right)$ and the parameters. We assume the so-called type I hybrid specification:

$$
\begin{aligned}
& \mathbf{e}_{t}=\mathbf{z}_{t} \mathbf{H}_{t}^{\frac{1}{2}} \sqrt{g_{t}}, \\
& \ln g_{t}=\phi \ln g_{t-1}+\sigma_{g} \eta_{t}, \\
& {\left[\mathbf{z}_{t}, \eta_{t}\right]^{\prime} \sim i i N\left(\mathbf{0}_{[(n+1) \times 1]}, \mathbf{I}_{n+1}\right),} \\
& \mathbf{H}_{t}=(1-\beta-\gamma) \mathbf{A}+\beta\left(\mathbf{e}_{t-1}^{\prime} \mathbf{e}_{t-1}\right)+\gamma \mathbf{H}_{t-1} .
\end{aligned}
$$

That is, $\mathbf{e}_{t}$ is conditionally Normal with mean vector $\mathbf{0}$ and covariance matrix $g_{t} \mathbf{H}_{t}$, where $g_{t}$ is a latent process and $\mathbf{H}_{t}$ is a square matrix of order $n$ that has the scalar $\operatorname{BEKK}(1,1)$ structure. Thus, the conditional distribution of $\mathbf{r}_{t}$ (given its past and latent variables) is Normal with mean $\mathbf{m}_{t}=\mathbf{d}_{0}+\mathbf{r}_{t-1} \boldsymbol{\Phi}$ and covariance matrix $g_{t} \mathbf{H}_{t}$.
The presence of the latent $\mathrm{AR}(1)$ process in the conditional covariance matrix helps in explaining outlying observations, and the dependence on the past data (through the SBEKK structure of $\mathbf{H}_{t}$ ) prevents the entries of the conditional covariance matrix $g_{t} \mathbf{H}_{t}$ from sharing the same dynamic pattern. Thus the model has time-varying conditional correlations without introducing more latent processes. In fact, the hybrid model defined by (12)-(14) nests two simple basic structures. In the limiting case when $\sigma_{g} \rightarrow 0$ and $\phi=0$ we are in the SBEKK model, while $\beta=0$ and $\gamma=0$ lead to the MSF case.

In (14) $\mathbf{A}$ is a free symmetric positive definite matrix of order $n$, with an inverted Wishart prior distribution (in fact, for $\mathbf{A}^{-1}$ we assume the Wishart prior with $n$ degrees of freedom and mean $\mathbf{I}_{n}$ ), and $\beta$ and $\gamma$ are free scalar parameters, jointly uniformly distributed over the unit simplex. As regards initial conditions for $\mathbf{H}_{t}$, we take $\mathbf{H}_{0}=h_{0} \mathbf{I}_{n}$ and treat $h_{0}>0$ as an additional parameter, a priori Exponentially distributed with mean 1 . For the parameters of the latent process we use the same priors as in [13]; for $\phi$ : Normal with mean 0 and variance 100 , truncated to $(-1,1)$, for $\sigma_{g}^{-2}$ : Exponential with mean $200 ; g_{0}$ is fixed (equals 1 ).

In order to obtain the required quantiles of the predictive distribution of future logarithmic returns, we follow the approximation explained in [9]. That is, we use OLS for the $\operatorname{VAR}(1)$ parameters and replace $\mathbf{A}$ by the empirical covariance matrix of the OLS residuals from the $\operatorname{VAR}(1)$ part. The Bayesian analysis for the remaining parameters and future return rates is then based on the conditional posterior and predictive distributions given the particular values of the highly dimensional parameters ( $\mathbf{d}$ and $\mathbf{A}$ ). These conditional distributions are sampled using the Gibbs scheme with Metropolis-Hastings steps, as shown in detail in [9].

In order to make the univariate model of portfolio value comparable to the $n$-variate volatility model of individual assets, we consider for the portfolio logarithmic returns the univariate $\operatorname{AR}(1)$ specification with the error term described by the hybrid SV-GARCH $(1,1)$ process, which is the $n=1$ special case of the MSF-SBEKK $(1,1)$ structure. So we assume

$$
\begin{aligned}
& r_{t}=\delta_{0}+\delta r_{t-1}+\varepsilon_{t}, \\
& \varepsilon_{t}=\zeta_{t} \sqrt{g_{t} h_{t}}, \\
& \ln g_{t}=\phi \ln g_{t-1}+\sigma_{g} \eta_{t}, \\
& {\left[\zeta_{t}, \eta_{t}\right]^{\prime} \sim i i N\left(\mathbf{0}_{[2 \times 1]}, \mathbf{I}_{2}\right),} \\
& h_{t}=(1-\beta-\gamma) \tilde{a}+\beta\left(\varepsilon_{t-1}\right)^{2}+\gamma h_{t-1}, \\
& \quad t=1, \ldots, T, \ldots, T+s .
\end{aligned}
$$

We take the prior distribution corresponding to the previous ( $n$-variate) case (now with $n=1$ ). Now we do not face the dimensionality problem, but for comparison with the $n$-variate model, the posterior and predictive distribution is sampled (using the Gibbs scheme with Metropolis-Hastings steps) conditionally on preliminary non-Bayesian (OLS) estimates of $\delta_{0}, \delta, \tilde{a}$ - as in the $n$ variate case. 


\section{Empirical results}

As the dataset we use the same stock data as in [11]; they represent 50 companies and cover the period May 13, 2005 - February 23, 2010. In February or March 2010 these companies were included in the two important indices of the Warsaw Stock Exchange, namely WIG20 and mWIG40. We start with $T=939$ initial observations (from the period May 13, 2005 - May, 12, 2009) and consider $p=200 \mathrm{VaR}$ and ES assessments for 1-, 2-, .., 10-day trading horizons. For Bayesian estimation the whole dataset available at time $T+k(k=0,1, \ldots, p-$ 1 ) is used. We calculate predictive distributions of $\mathbf{r}_{t}$ based on the dataset available at time $T+k$ for each $k=$ $0,1, \ldots, p-1$ (up to $T+p-1=1138$ ). Thus we obtain 200 distributions for 1-, 2-, .., 10-day forecast horizons, and then $\operatorname{VaR}_{t: t+1}^{j}(\alpha)$ and $\operatorname{ES}_{t: t+1}^{j}(\alpha)$ for $j \in\{L, S\}$ and
$t=T, \ldots, T+p-1$.

We consider two portfolios: one consisting of one unit of each asset, i.e. $\mathbf{a}=\left[a_{1}, a_{2}, \ldots, a_{n}\right]=[1, \ldots, 1]$ and the other of $a_{\tau, i}=\frac{1}{S_{\tau, i}}\left[\frac{1}{n} \sum_{j=1}^{n} S_{\tau, j}\right]$ units of asset $i$, that is $\omega_{\tau, i}=1 / 50$, where $i=1, \ldots, 50$, and $\tau$ represents May $12,2009$.

In order to compare one period ahead VaR and ES obtained in two different ways, i.e. using $n$-variate MSF-SBEKK model for individual assets or its univariate counterpart for the portfolio value, we use popular non-Bayesian criteria. They include the failure rate and $p$-value for the Kupiec test as well as the "tick" and the Lopez loss functions (defined below); see Tables I-IV . For the sake of comparison we also use some non-parametric models, specially designed for direct VaR or ES assessment. In particular, we apply the CAViaR model with asymmetric slope:

$q_{t}(\alpha)=\beta_{0}+\beta_{1} q_{t-1}(\alpha)+\beta_{2}\left|D_{t-2: t-1}\right|+\beta_{3}\left|D_{t-2: t-1}\right| I_{(-\infty, 0)}\left(D_{t-2: t-1}\right)$

of [2]; it is applied directly to the series $\left\{D_{t: t+s}\right\}$ of daily value changes $D_{t: t+s}=W_{t+s}-W_{t}$ (not to the logarithmic returns); thus, $q_{t}(\alpha)$ denotes the conditional $\alpha$-quantile of $D_{t-1: t}, I_{(-\infty, 0)}(\cdot)$ is the characteristic function of the interval $(-\infty, 0)$. We also use the CARE model with asymmetric slope:

$$
\mu_{t}(\tau)=\gamma_{0}+\gamma_{1} \mu_{t-1}(\tau)+\gamma_{2}\left|D_{t-2: t-1}\right|+\gamma_{3}\left|D_{t-2: t-1}\right| I_{(-\infty, 0)}\left(D_{t-2: t-1}\right),
$$

where $\mu_{t}(\tau)$ is the conditional $\tau$ expectile, i.e.

$$
\mu_{t}(\tau)=\underset{\mu}{\operatorname{argmin}} E\left[\left|\tau-I_{(-\infty, 0)}\left(D_{t-1: t}-\mu\right)\right|\left(D_{t-1: t}-\mu\right)^{2} \mid \Psi_{t-1}\right] .
$$

Given $\tau$, the best choice of $\gamma=\left[\gamma_{0}, \gamma_{1}, \gamma_{2}, \gamma_{3}\right]^{\prime}$ is:

$$
\widehat{\gamma}=\underset{\gamma}{\operatorname{argmin}} \sum_{t=1}^{T}\left|\tau-I_{(-\infty, 0)}\left(D_{t-1: t}-\mu_{t}(\tau)\right)\right|\left[D_{t-1: t}-\mu_{t}(\tau)\right]^{2} .
$$

Using conditional expectiles we estimate the Expected Shortfall by:

$$
\operatorname{ES}_{t: t+1}^{L}(\alpha)=-\left[1+\frac{\tau}{(1-2 \tau) \alpha}\right] \mu_{t}(\alpha)+\left[\frac{\tau}{(1-2 \tau) \alpha}\right] E\left(D_{t-1: t} \mid \Psi_{t-1}\right),
$$

see [5]. Note that in the case of $E\left(D_{t-1: t} \mid \Psi_{t-1}\right)=0$, inserting $\mu_{t}(\alpha)$ from (20) into (23) gives the following equality:

$$
\operatorname{ES}_{t: t+1}^{L}(\alpha)=\lambda_{0}+\lambda_{1} \operatorname{ES}_{t-1: t}^{L}(\alpha)+\lambda_{2}\left|D_{t-2: t-1}\right|+\lambda_{3}\left|D_{t-2: t-1}\right| I_{(-\infty, 0)}\left(D_{t-2: t-1}\right),
$$

where $\lambda_{1}=\gamma_{1}, \lambda_{i}=-\left[1+\frac{\tau}{(1-2 \tau) \alpha}\right] \gamma_{i}, i=0,2,3$.

To obtain ES for a short trading position we apply (23) to the series of daily value changes multiplied by minus one (i.e. $\left\{-D_{t: t+s}\right\}$, for details see [5]).

The losses are generally calculated as $L_{s}^{j}=\frac{1}{p} \sum_{t=T}^{T+p-1} l_{t: t+s}^{j}$, where for $l_{t: t+s}^{j}$ and $j \in\{L, S\}$ we have

- the "tick" loss if

$$
\begin{aligned}
& l_{t: t+s}^{L}= \begin{cases}(\alpha-1)\left[D_{t: t+s}+\operatorname{VaR}_{t: t+s}^{L}(\alpha)\right], & \text { if } D_{t: t+s}<-\operatorname{VaR}_{t: t+s}^{L}(\alpha), \\
\alpha\left[D_{t: t+s}+\operatorname{VaR}_{t: t+s}^{L}(\alpha)\right], & \text { if } D_{t: t+s} \geq-\operatorname{VaR}_{t: t+s}^{L}(\alpha) ;\end{cases} \\
& l_{t: t+s}^{S}= \begin{cases}(\alpha-1)\left[D_{t: t+s}-\operatorname{VaR}_{t: t+s}^{S}(\alpha)\right], & \text { if } D_{t: t+s}>\operatorname{VaR}_{t: t+s}^{S}(\alpha), \\
\alpha\left[D_{t: t+s}-\operatorname{VaR}_{t: t+s}^{S}(\alpha)\right], & \text { if } D_{t: t+s} \leq \operatorname{VaR}_{t: t+s}^{S}(\alpha) ;\end{cases}
\end{aligned}
$$

- the Lopez loss if

$$
l_{t: t+s}^{L}= \begin{cases}1+\left[D_{t: t+s}+\operatorname{VaR}_{t: t+s}^{L}(\alpha)\right]^{2}, & \text { if } D_{t: t+s}<-\operatorname{VaR}_{t: t+s}^{L}(\alpha) \\ 0, & \text { if } D_{t: t+s} \geq-\operatorname{VaR}_{t: t+s}^{L}(\alpha)\end{cases}
$$




$$
l_{t: t+s}^{S}= \begin{cases}1+\left[D_{t: t+s}-\operatorname{VaR}_{t: t+s}^{S}(\alpha)\right]^{2}, & \text { if } D_{t: t+s}>\operatorname{VaR}_{t: t+s}^{S}(\alpha) \\ 0, & \text { if } D_{t: t+s} \leq \operatorname{VaR}_{t: t+s}^{S}(\alpha)\end{cases}
$$

see e.g. [14-16].

For backtesting the forecasted Expected Shortfall we use different measures proposed by Zhu and Galbraith [17], Kaufmann and Patie [18], and Embrechts, Kaufmann and Patie [19]. We compute the mean error:

$$
M E_{s}^{j}(\alpha)=\operatorname{ES}_{s}^{A, j}(\alpha)-A L_{s}^{j}(\alpha)
$$

where $j \in\{L, S\}, \operatorname{ES}_{s}^{A, j}(\alpha)$ is the average predictive ES:

$$
\begin{aligned}
& \operatorname{ES}_{s}^{A, L}(\alpha)=\frac{1}{J_{s}^{L}} \sum_{t=T}^{T+p-1} I_{(-\infty, 0)}\left[D_{t: t+s}+\operatorname{VaR}_{t: t+s}^{L}(\alpha)\right] \operatorname{ES}_{t: t+s}^{L}(\alpha), \\
& \operatorname{ES}_{s}^{A, S}(\alpha)=\frac{1}{J_{s}^{S}} \sum_{t=T}^{T+p-1} I_{(-\infty, 0)}\left[D_{t: t+s}-\operatorname{VaR}_{t: t+s}^{S}(\alpha)\right] \operatorname{ES}_{t: t+s}^{S}(\alpha),
\end{aligned}
$$

$A L_{s}^{j}(\alpha)$ is the average loss on the portfolio when the loss is larger than $\operatorname{VaR}_{t: t+s}^{j}(\alpha)$ :

$$
\begin{aligned}
& A L_{s}^{L}(\alpha)=\frac{1}{J_{s}^{L}} \sum_{t=T}^{T+p-1} I_{(-\infty, 0)}\left[D_{t: t+s}+\operatorname{VaR}_{t: t+s}^{L}(\alpha)\right]\left|D_{t: t+s}\right|, \\
& A L_{s}^{S}(\alpha)=\frac{1}{J_{s}^{S}} \sum_{t=T}^{T+p-1} I_{(-\infty, 0)}\left[D_{t: t+s}-\operatorname{VaR}_{t: t+s}^{S}(\alpha)\right]\left|D_{t: t+s}\right| \\
& J_{s}^{L}=\sum_{t=T}^{T+p-1} I_{(-\infty, 0)}\left[D_{t: t+s}+\operatorname{VaR}_{t: t+s}^{L}(\alpha)\right] \\
& J_{s}^{S}=\sum_{t=T}^{T+p-1} I_{(-\infty, 0)}\left[D_{t: t+s}-\operatorname{VaR}_{t: t+s}^{S}(\alpha)\right]
\end{aligned}
$$

As Zhu and Galbraith [17] have noticed, if the average predictive ES is lower (higher) than $A L_{s}^{j}$ ("observed ES"), then the model tends to underestimate (overestimate) risk. A good forecast of ES will lead to a low absolute value of the mean error. Another measure of predictive out-of-sample performance for ES is the mean absolute error:

$$
M A E_{s}^{L}(\alpha)=\frac{1}{J_{s}^{L}} \sum_{t=T}^{T+p-1} I_{(-\infty, 0)}\left[D_{t: t+s}+\operatorname{VaR}_{t: t+s}^{L}(\alpha)\right]\left|A L_{s}^{L}(\alpha)-\operatorname{ES}_{t: t+s}^{L}(\alpha)\right|,
$$

for a long trading position;

$$
M A E_{s}^{S}(\alpha)=\frac{1}{J_{s}^{S}} \sum_{t=T}^{T+p-1} I_{(-\infty, 0)}\left[D_{t: t+s}-\operatorname{VaR}_{t: t+s}^{S}(\alpha)\right]\left|A L_{s}^{S}(\alpha)-\operatorname{ES}_{t: t+s}^{S}(\alpha)\right|,
$$

for a short trading position.

Also, the mean absolute percentage error can be considered:

$$
M A P E_{s}^{L}(\alpha)=\frac{1}{J_{s}^{L}} \sum_{t=T}^{T+p-1} I_{(-\infty, 0)}\left[D_{t: t+s}+\operatorname{VaR}_{t: t+s}^{L}(\alpha)\right] \frac{\left|A L_{s}^{L}(\alpha)-\operatorname{ES}_{t: t+s}^{L}(\alpha)\right|}{\left|A L_{s}^{L}(\alpha)\right|},
$$

for a long trading position;

$$
M A P E_{s}^{S}(\alpha)=\frac{1}{J_{s}^{S}} \sum_{t=T}^{T+p-1} I_{(-\infty, 0)}\left[D_{t: t+s}-\operatorname{VaR}_{t: t+s}^{S}(\alpha)\right] \frac{\left|A L_{s}^{S}(\alpha)-\operatorname{ES}_{t: t+s}^{S}(\alpha)\right|}{\left|A L_{s}^{S}(\alpha)\right|},
$$

for a short trading position.

We also compute the backtesting measures proposed by Kaufmann and Patie [18]:

$$
\begin{aligned}
& V_{1}^{\mathrm{ES}, L}(\alpha)=\frac{1}{J_{1}^{L}} \sum_{t=T}^{T+p-1} I_{(-\infty, 0)}\left[D_{t: t+s}+\operatorname{VaR}_{t: t+s}^{L}(\alpha)\right]\left[D_{t: t+1}+\operatorname{ES}_{t: t+1}^{L}(\alpha)\right], \\
& V_{1}^{\mathrm{ES}, S}(\alpha)=\frac{1}{J_{1}^{S}} \sum_{t=T}^{T+p-1} I_{(-\infty, 0)}\left[D_{t: t+s}-\operatorname{VaR}_{t: t+s}^{S}(\alpha)\right]\left[D_{t: t+1}-\operatorname{ES}_{t: t+1}^{S}(\alpha)\right],
\end{aligned}
$$




$$
\begin{aligned}
V_{2}^{\mathrm{ES}, L}(\alpha) & =\frac{\sum_{t=T}^{T+p-1} I_{(-\infty, 0)}\left(D_{t: t+s}-D^{\alpha, L}\right)\left[D_{t: t+1}+\mathrm{ES}_{t: t+1}^{L}(\alpha)\right]}{\sum_{t=T}^{T+p-1} I_{(-\infty, 0)}\left(D_{t: t+s}-D^{\alpha, L}\right)}, \\
V_{2}^{\mathrm{ES}, S}(\alpha) & =\frac{\sum_{t=T}^{T+p-1} I_{(-\infty, 0)}\left(D_{t: t+s}-D^{\alpha, S}\right)\left[D_{t: t+1}-\mathrm{ES}_{t: t+1}^{S}(\alpha)\right]}{\sum_{t=T}^{T+p-1} I_{(-\infty, 0)}\left(D_{t: t+s}-D^{\alpha, S}\right)},
\end{aligned}
$$

where $D^{\alpha, L}$ and $D^{\alpha, S}$ are the empirical $\alpha$-quantiles of $\left[D_{t: t+1}+\operatorname{ES}_{t: t+1}^{L}(\alpha)\right]$ and $\left[D_{t: t+1}-\operatorname{ES}_{t: t+1}^{S}(\alpha)\right]$, respectively, $t=T, \ldots, T+p-1$. A good prediction of ES will lead to low absolute values of $V_{1}^{\mathrm{ES}, j}(\alpha), V_{2}^{\mathrm{ES}, j}(\alpha)$ and $V^{\mathrm{ES}, j}(\alpha)$. Note that $V_{1}^{\mathrm{ES}, L}(\alpha)=M E_{1}^{L}(\alpha)$ and $V_{1}^{\mathrm{ES}, S}(\alpha)=-M E_{1}^{S}(\alpha)$. Moreover, $V_{1}^{\mathrm{ES}, j}(\alpha)$ strongly depends on VaR assessments, because it considers only values of $D_{t: t+1}$ which exceed this threshold.

\subsection{1-day risk measures assessments}

The outcomes of the Kupiec test for one period ahead VaR seem to indicate that our Bayesian assessment (based on the parametric MSF-SBEKK structure) competes with the one based on CAViaR (Table II; the best

TABLE I

The failure rate for $\operatorname{VaR}_{t: t+1}^{L}(\alpha)$ and $\operatorname{VaR}_{t: t+1}^{S}(\alpha)$. Here and further PF1 is Portfolio with $\mathbf{a}=[1, \ldots, 1]^{\prime}$, $\mathrm{PF} 2$ - Portfolio with $\omega_{\tau, i}=1 / 50, \mathrm{AA}-n$-variate MSF-SBEKK, BB - univariate SV-GARCH, CC -

\begin{tabular}{|c|c|c|c|c|c|c|}
\hline \multirow[t]{2}{*}{$\alpha$} & \multicolumn{3}{|c|}{ PF1 } & \multicolumn{3}{|c|}{ PF2 } \\
\hline & AA & $\mathrm{BB}$ & $\mathrm{CC}$ & AA & $\mathrm{BB}$ & $\mathrm{CC}$ \\
\hline \multicolumn{7}{|c|}{ long trading position } \\
\hline 0.01 & 0.010 & 0.015 & 0.015 & 0.010 & 0.020 & 0.010 \\
\hline 0.025 & 0.020 & 0.025 & 0.030 & 0.020 & 0.025 & 0.025 \\
\hline 0.05 & 0.045 & 0.065 & 0.060 & 0.055 & 0.040 & 0.035 \\
\hline 0.1 & 0.110 & 0.120 & 0.105 & 0.090 & 0.100 & 0.090 \\
\hline \multicolumn{7}{|c|}{ short trading position } \\
\hline 0.1 & 0.115 & 0.145 & 0.125 & 0.110 & 0.105 & 0.070 \\
\hline 0.05 & 0.065 & 0.100 & 0.075 & 0.050 & 0.045 & 0.030 \\
\hline 0.025 & 0.045 & 0.070 & 0.040 & 0.020 & 0.015 & 0.010 \\
\hline 0.01 & 0.010 & 0.020 & 0.010 & 0.000 & 0.010 & 0.000 \\
\hline
\end{tabular}
CAViaR

The failure rate is defined as the proportion of $D_{t: t+1}$ 's smaller than the $-\operatorname{VaR}_{t: t+1}^{L}(\alpha)$ or the proportion of $D_{t: t+1}$ 's bigger than the $\operatorname{VaR}_{t: t+1}^{S}(\alpha)$

case is in bold). Which model is better depends on the particular criterion (see Table I-X). For example, for $\operatorname{VaR}_{t: t+1}^{L}(\alpha)$ the "tick" loss function points at the $n$ variate MSF-SBEKK model, while the Lopez loss function suggests that CAViaR is the optimal model (except $\alpha$ equal to 0.05 or 0.1 for long trading positions, see Table III and IV). The results show that the $n$-variate
TABLE II

The $p$-value for the Kupiec test for $\operatorname{VaR}_{t: t+1}^{L}(\alpha)$ and $\operatorname{VaR}_{t: t+1}^{S}(\alpha)$

\begin{tabular}{ccc|c|ccc}
\hline \hline \multirow{2}{*}{$\alpha$} & \multicolumn{3}{c|}{ PF1 } & \multicolumn{3}{c}{ PF2 } \\
\cline { 2 - 6 } & AA & BB & CC & AA & BB & CC \\
\hline \multicolumn{6}{c}{ long trading position } \\
\hline 0.01 & $\mathbf{1 . 0 0 0}$ & 0.508 & 0.508 & $\mathbf{1 . 0 0 0}$ & 0.211 & $\mathbf{1 . 0 0 0}$ \\
0.025 & 0.639 & $\mathbf{1 . 0 0 0}$ & 0.660 & 0.639 & $\mathbf{1 . 0 0 0}$ & $\mathbf{1 . 0 0 0}$ \\
0.05 & $\mathbf{0 . 7 4 2}$ & 0.351 & 0.529 & $\mathbf{0 . 7 4 9}$ & 0.502 & 0.305 \\
0.1 & 0.642 & 0.359 & $\mathbf{0 . 8 1 5}$ & 0.632 & $\mathbf{1 . 0 0 0}$ & $\mathbf{0 . 6 3 2}$ \\
\hline \multicolumn{7}{c}{ short trading position } \\
\hline 0.1 & $\mathbf{0 . 4 8 9}$ & 0.045 & 0.255 & 0.642 & $\mathbf{0 . 8 1 5}$ & 0.137 \\
0.05 & $\mathbf{0 . 3 5 1}$ & 0.004 & 0.130 & $\mathbf{1 . 0 0 0}$ & 0.742 & 0.162 \\
0.025 & 0.103 & 0.001 & $\mathbf{0 . 2 1 1}$ & $\mathbf{0 . 6 3 9}$ & 0.328 & 0.123 \\
0.01 & $\mathbf{1 . 0 0 0}$ & 1.211 & $\mathbf{1 . 0 0 0}$ & 0.045 & $\mathbf{1 . 0 0 0}$ & 0.045
\end{tabular}

TABLE III

"Tick" loss function for $\operatorname{VaR}_{t: t+1}^{L}(\alpha)$ and $\operatorname{VaR}_{t: t+1}^{S}(\alpha)$

\begin{tabular}{cccc|ccc}
\hline \hline \multirow{2}{*}{$\alpha$} & \multicolumn{3}{c|}{ PF1 } & \multicolumn{3}{c}{ PF2 } \\
\cline { 2 - 6 } & AA & BB & CC & AA & BB & CC \\
\hline \multicolumn{6}{c}{ long trading position } \\
\hline 0.01 & $\mathbf{2 . 2 8 6}$ & 2.341 & 2.430 & 1.991 & 2.084 & $\mathbf{1 . 9 7 1}$ \\
0.025 & $\mathbf{4 . 3 8 9}$ & 4.509 & 4.902 & $\mathbf{3 . 9 4 2}$ & 4.258 & 4.210 \\
0.05 & $\mathbf{7 . 4 3 1}$ & 7.581 & 8.018 & $\mathbf{6 . 7 2 9}$ & 6.894 & 7.211 \\
0.1 & $\mathbf{1 2 . 3 2 4}$ & 12.427 & 12.920 & 11.139 & $\mathbf{1 0 . 9 9 6}$ & 11.464 \\
\hline \multicolumn{6}{c}{ short trading position } \\
\hline 0.1 & 12.461 & 12.100 & $\mathbf{1 1 . 5 1 0}$ & 10.456 & $\mathbf{9 . 8 1 4}$ & 9.913 \\
0.05 & 7.515 & 7.573 & $\mathbf{6 . 7 0 9}$ & 5.970 & 5.970 & $\mathbf{5 . 9 4 0}$ \\
0.025 & 4.230 & 4.122 & $\mathbf{3 . 6 9 5}$ & $\mathbf{3 . 3 9 1}$ & 3.437 & 3.526 \\
0.01 & 1.820 & 1.891 & $\mathbf{1 . 5 7 2}$ & $\mathbf{1 . 5 9 8}$ & 1.636 & 1.704
\end{tabular}

MSF-SBEKK approach is helpful for portfolio risk assessment. The failure rates show that, for the portfolio with one unit of each asset, VaR tends to be underestimated in the univariate models (see Table I). In other words, the chance of a daily change in portfolio value being below $-\operatorname{VaR}_{t: t+1}^{L}(\alpha)$ (or above $\operatorname{VaR}_{t: t+1}^{S}(\alpha)$ ), which should be $\alpha$, is in fact greater.

Interestingly, the univariate SV-GARCH specification leads to VaR assessments that are highly correlated with the ones based on CAViaR (see Table V and Fig. 1). 
TABLE IV Lopez loss function for $\operatorname{VaR}_{t: t+1}^{L}(\alpha)$ and $\operatorname{VaR}_{t: t+1}^{S}(\alpha)$

\begin{tabular}{cccc|ccc}
\hline \hline \multirow{2}{*}{$\alpha$} & \multicolumn{3}{c|}{ PF1 } & \multicolumn{3}{c}{ PF2 } \\
\cline { 2 - 7 } & AA & BB & CC & AA & BB & CC \\
\hline \multicolumn{6}{c}{ long trading position } \\
\hline 0.01 & 63.316 & 68.848 & $\mathbf{1 9 . 5 2 3}$ & 20.691 & 18.022 & $\mathbf{6 . 4 0 0}$ \\
0.025 & 112.060 & 133.847 & $\mathbf{9 9 . 8 0 8}$ & 52.341 & 71.158 & $\mathbf{1 3 . 5 3 1}$ \\
0.05 & $\mathbf{1 8 7 . 1 0 8}$ & 227.417 & 210.195 & 106.337 & 146.965 & $\mathbf{8 5 . 3 0 6}$ \\
0.1 & $\mathbf{3 4 8 . 8 8 8}$ & 416.769 & 366.585 & 241.865 & 284.189 & $\mathbf{2 2 8 . 0 2 7}$ \\
\hline \multicolumn{6}{c}{ short trading position } \\
\hline 0.1 & 301.502 & 333.001 & $\mathbf{1 7 3 . 8 2 7}$ & 128.890 & 144.762 & $\mathbf{8 9 . 1 6 3}$ \\
0.05 & 114.033 & 145.079 & $\mathbf{5 9 . 7 0 7}$ & 30.730 & 46.040 & $\mathbf{1 3 . 4 0 4}$ \\
0.025 & 31.434 & 54.677 & $\mathbf{2 2 . 5 1 2}$ & 3.936 & 12.397 & $\mathbf{2 . 4 0 4}$ \\
0.01 & 2.679 & 12.165 & $\mathbf{2 . 4 9 1}$ & $\mathbf{0 . 0 0 0}$ & 0.332 & $\mathbf{0 . 0 0 0}$
\end{tabular}

TABLE V

Correlation coefficients between $\operatorname{VaR}_{t: t+1}^{j}(\alpha)$ for for different methods of assessment, $\omega_{\tau, i}=1 / 50$

\begin{tabular}{l|l|l|l|l|l}
\hline \hline$\alpha$ & 0.01 & 0.025 & 0.05 & 0.1 \\
\hline \multicolumn{5}{c}{ long trading position } \\
\hline $\begin{array}{l}\text { Corr }(n \text {-variate MSF-SBEKK, } \\
\text { univ. SV-GARCH) }\end{array}$ & 0.362 & 0.355 & 0.372 & 0.402 \\
\hline $\begin{array}{l}\text { Corr }(n \text {-variate MSF-SBEKK, } \\
\text { CAViaR) }\end{array}$ & 0.264 & 0.276 & 0.247 & 0.247 \\
\hline $\begin{array}{l}\text { Corr(univ. SV-GARCH, } \\
\text { CAViaR) }\end{array}$ & $\mathbf{0 . 9 0 2}$ & $\mathbf{0 . 8 2 7}$ & $\mathbf{0 . 8 5 7}$ & $\mathbf{0 . 7 6 5}$ \\
\hline $\begin{array}{l}\text { Corr }(n \text {-variate MSF-SBEKK, } \\
\text { univ. SV-GARCH) }\end{array}$ & 0.446 & 0.447 & 0.458 & 0.489 \\
\hline $\begin{array}{l}\text { Corr }(n \text {-variate MSF-SBEKK, } \\
\text { CAViaR) }\end{array}$ & 0.286 & 0.267 & 0.272 & 0.256 \\
\hline $\begin{array}{l}\text { Corr }(u n i v . \\
\text { CAViaR) }\end{array}$ & SV-GARCH, & $\mathbf{0 . 9 0 5}$ & $\mathbf{0 . 9 0 9}$ & $\mathbf{0 . 8 7 7}$ & $\mathbf{0 . 8 1 3}$ \\
\hline
\end{tabular}

TABLE VI

Mean errors for $\operatorname{ES}_{t: t+1}^{L}(\alpha)$ and $\operatorname{ES}_{t: t+1}^{S}(\alpha)$. Here and further DD denotes CAViaR CARE

\begin{tabular}{cccc|ccc}
\hline \hline \multirow{2}{*}{$\alpha}$. & \multicolumn{3}{c|}{ PF1 } & \multicolumn{3}{c}{ PF2 } \\
\cline { 2 - 6 } & AA & BB & DD & AA & BB & DD \\
\hline \multicolumn{6}{c}{ long trading position } \\
\hline 0.01 & -20.580 & -19.185 & $\mathbf{6 . 6 8 7}$ & -13.510 & $\mathbf{1 2 . 1 7 9}$ & 45.473 \\
0.025 & -9.300 & -19.471 & $\mathbf{- 9 . 2 0 4}$ & $\mathbf{- 9 . 9 1 3}$ & -12.015 & 40.514 \\
0.05 & -2.719 & -4.154 & $\mathbf{- 1 . 1 6 3}$ & $\mathbf{3 . 7 8 1}$ & -13.777 & 26.369 \\
0.1 & $\mathbf{2 . 8 3 5}$ & -5.463 & -3.158 & -4.931 & $\mathbf{- 1 . 4 4 2}$ & 27.076 \\
\hline \multicolumn{6}{c}{ short trading position } \\
\hline 0.1 & -4.397 & -5.173 & $\mathbf{2 . 2 1 1}$ & $\mathbf{8 . 4 4 1}$ & 9.678 & 9.808 \\
0.05 & 2.301 & $\mathbf{1 . 1 7 2}$ & 5.560 & 16.209 & $\mathbf{1 0 . 1 2 4}$ & 14.812 \\
0.025 & 14.378 & 13.674 & 11.652 & 19.159 & $\mathbf{3 . 8 0 3}$ & 20.692 \\
0.01 & 24.171 & 11.917 & 25.647 & - & 28.629 & -
\end{tabular}

TABLE VII

Mean absolute errors for $\operatorname{ES}_{t: t+1}^{L}(\alpha)$ and $\operatorname{ES}_{t: t+1}^{S}(\alpha)$

\begin{tabular}{cccc|ccc}
\hline \hline \multirow{2}{*}{$\alpha$} & \multicolumn{3}{c|}{ PF1 } & \multicolumn{3}{c}{ PF2 } \\
\cline { 2 - 6 } & AA & BB & DD & AA & BB & DD \\
\hline \multicolumn{6}{c}{ long trading position } \\
\hline 0.01 & 52.327 & $\mathbf{3 9 . 1 9 3}$ & 42.362 & $\mathbf{3 7 . 2 7 9}$ & 39.951 & 56.932 \\
0.025 & 25.764 & 25.778 & $\mathbf{1 6 . 2 7 7}$ & 62.078 & $\mathbf{3 9 . 0 9 5}$ & 48.256 \\
0.05 & 39.660 & 28.252 & $\mathbf{2 4 . 1 9 7}$ & 37.377 & $\mathbf{3 1 . 0 1 8}$ & 31.456 \\
0.1 & 30.316 & 23.212 & $\mathbf{2 3 . 9 3 3}$ & 29.027 & $\mathbf{1 8 . 1 7 0}$ & 29.720 \\
\hline \multicolumn{6}{c}{ short trading position } \\
\hline 0.1 & 24.229 & 18.383 & $\mathbf{1 5 . 8 2 2}$ & 26.212 & $\mathbf{1 8 . 8 9 8}$ & 20.417 \\
0.05 & 24.047 & $\mathbf{1 6 . 6 0 4}$ & 19.487 & 27.287 & $\mathbf{2 1 . 5 2 2}$ & 21.724 \\
0.025 & 22.954 & 22.636 & $\mathbf{2 2 . 5 0 4}$ & 29.327 & $\mathbf{1 0 . 2 0 2}$ & 20.692 \\
0.01 & 39.331 & $\mathbf{2 5 . 8 7 5}$ & 47.553 & - & 28.629 & -
\end{tabular}

TABLE VIII

Mean absolute percentage errors for $\operatorname{ES}_{t: t+1}^{L}(\alpha)$ and $\operatorname{ES}_{t: t+1}^{S}(\alpha)$

\begin{tabular}{|c|c|c|c|c|c|c|}
\hline \multirow[t]{2}{*}{$\alpha$} & \multicolumn{3}{|c|}{ PF1 } & \multicolumn{3}{|c|}{$\mathrm{PF} 2$} \\
\hline & AA & $\mathrm{BB}$ & DD & $\mathrm{AA}$ & $\mathrm{BB}$ & DD \\
\hline \multicolumn{7}{|c|}{ long trading position } \\
\hline 0.01 & 0.245 & 0.208 & 0.196 & 0.297 & 0.232 & 0.376 \\
\hline 0.025 & 0.145 & 0.152 & 0.102 & 0.400 & 0.250 & 0.310 \\
\hline 0.05 & 0.270 & 0.212 & 0.179 & 0.305 & 0.220 & 0.220 \\
\hline 0.1 & 0.276 & 0.210 & 0.212 & 0.278 & 0.178 & 0.278 \\
\hline \multicolumn{7}{|c|}{ short trading position } \\
\hline 0.1 & 0.224 & 0.180 & 0.144 & 0.279 & 0.190 & 0.182 \\
\hline 0.05 & 0.204 & 0.145 & 0.160 & 0.271 & 0.182 & 0.176 \\
\hline 0.025 & 0.189 & 0.188 & 0.168 & 0.168 & 0.087 & 0.159 \\
\hline 0.01 & 0.254 & 0.177 & 0.286 & - & 0.220 & - \\
\hline
\end{tabular}

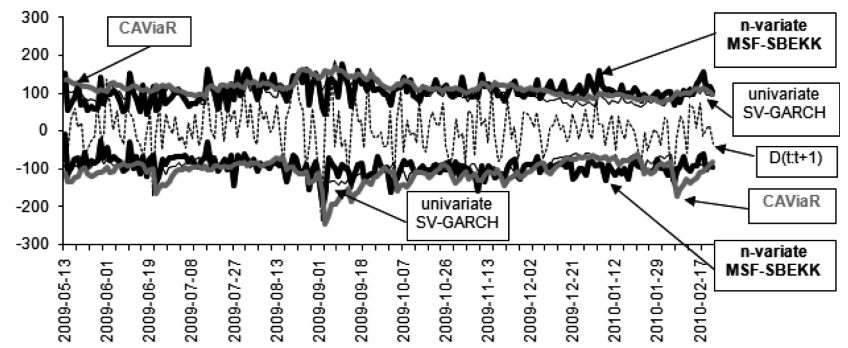

Fig. 1. $\operatorname{VaR}_{t: t+1}^{j}(0.05)$ results, $\omega_{\tau, i}=1 / 50, j \in\{L, S\}$. 
TABLE IX

$V^{\mathrm{ES}, j}(\alpha)$ for $\operatorname{ES}_{t: t+1}^{L}(\alpha)$ and $\operatorname{ES}_{t: t+1}^{S}(\alpha)$

\begin{tabular}{cccc|ccc}
\hline \hline \multirow{2}{*}{$\alpha$} & \multicolumn{3}{c|}{ PF1 } & \multicolumn{3}{c}{ PF2 } \\
\cline { 2 - 6 } & AA & BB & DD & AA & BB & DD \\
\hline \multicolumn{6}{c}{ long trading position } \\
\hline 0.01 & 20.580 & 19.185 & $\mathbf{6 . 6 2 5}$ & 13.510 & $\mathbf{1 2 . 1 7 9}$ & 33.465 \\
0.025 & $\mathbf{6 . 0 3 1}$ & 19.564 & 22.371 & $\mathbf{5 . 8 2 8}$ & 12.015 & 36.201 \\
0.05 & $\mathbf{2 . 1 0 6}$ & 9.042 & 19.127 & $\mathbf{2 . 2 8 3}$ & 8.731 & 32.759 \\
0.1 & $\mathbf{1 . 9 3 7}$ & 8.956 & 19.856 & 3.126 & $\mathbf{1 . 5 3 2}$ & 28.752 \\
\hline \multicolumn{6}{c}{ short trading position } \\
\hline 0.1 & $\mathbf{6 . 9 5 4}$ & 12.837 & 13.104 & $\mathbf{7 . 0 1 6}$ & 8.654 & 14.380 \\
0.05 & $\mathbf{4 . 3 8 4}$ & 7.604 & 11.073 & 15.481 & $\mathbf{1 1 . 2 7 8}$ & 20.098 \\
0.025 & $\mathbf{8 . 2 9 0}$ & 12.219 & 17.507 & 20.860 & $\mathbf{1 2 . 9 9 2}$ & 28.689 \\
0.01 & 24.171 & $\mathbf{6 . 0 6 6}$ & 31.836 & - & 28.629 & -
\end{tabular}

TABLE X

Correlation coefficients between $\operatorname{ES}_{t: t+1}^{j}(\alpha)$ for different methods of assessment, $\omega_{\tau, i}=1 / 50$

\begin{tabular}{|c|c|c|c|c|}
\hline$\alpha$ & 0.01 & 0.025 & 0.05 & 0.1 \\
\hline \multicolumn{5}{|c|}{ long trading position } \\
\hline $\begin{array}{l}\text { Corr }(n \text {-variate MSF-SBEKK, } \\
\text { univ. SV-GARCH) }\end{array}$ & 0.372 & 0.363 & 0.360 & 0.366 \\
\hline $\begin{array}{l}\operatorname{Corr}(n \text {-variate MSF-SBEKK, } \\
\text { CAViaR/CARE) }\end{array}$ & 0.185 & 0.205 & 0.209 & 0.232 \\
\hline $\begin{array}{l}\text { Corr(univ. SV-GARCH, } \\
\text { CAViaR/CARE) }\end{array}$ & 0.848 & 0.928 & 0.888 & 0.801 \\
\hline \multicolumn{5}{|c|}{ short trading position } \\
\hline $\begin{array}{l}\text { Corr }(n \text {-variate MSF-SBEKK, } \\
\text { univ. SV-GARCH) }\end{array}$ & 0.430 & 0.439 & 0.444 & 0.454 \\
\hline $\begin{array}{l}\operatorname{Corr}(n \text {-variate MSF-SBEKK, } \\
\text { CAViaR/CARE) }\end{array}$ & 0.297 & 0.298 & 0.302 & 0.304 \\
\hline $\begin{array}{l}\text { Corr(univ. SV-GARCH, } \\
\text { CAViaR/CARE) }\end{array}$ & 0.919 & 0.921 & 0.910 & 0.883 \\
\hline $\left.\begin{array}{l}300 \\
200\end{array}\right]$ & & & & $\begin{array}{l}\text { n-variate } \\
\text { ISF-SBEKK }\end{array}$ \\
\hline & & & & \\
\hline & & & & \\
\hline 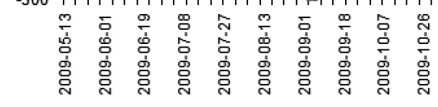 & 管 & 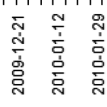 & dٌ & $\begin{array}{l}\text { univariate } \\
\text { SV-GARCH }\end{array}$ \\
\hline
\end{tabular}

Fig. 2. $\operatorname{ES}_{t: t+1}^{j}(0.05)$ results, $\omega_{\tau, i}=1 / 50, j \in\{L, S\}$.

Mean errors suggest that our parametric models (based on MSF-SBEKK structure) tend to underestimate ES for long trading positions, while CARE model frequently overestimates it (see Table VI). Mean absolute errors and mean absolute percentage errors for the 1-day ES indicate that the univariate approaches (SV-GARCH and CARE) are frequently better (see Table VII and VIII). Similarly to VaR assessments, the univariate SV-GARCH specification leads to ES assessments that are highly correlated with the ones based on CARE (see Table X and Fig. 2).

\section{2. s-day risk measures assessments}

We also consider the $s$-period ahead VaR and ES for larger $s$. Generally, our empirical findings obtained for the portfolio with one unit of each asset are similar to the ones based on the portfolio with $\omega_{\tau, i}=1 / 50$ (i.e. the main conclusions are the same).

The Lopez loss function values indicate that the $n$ variate MSF-BEKK model estimates VaR better than the univariate SV-GARCH model (see Tables XI and XII). As regards the ES assessment, the mean absolute errors and mean absolute percentage errors indicate that the univariate approach is frequently better or enough (expecially for short trading positions).

\section{Concluding remarks}

The aim of the paper was to compare the $n$-variate and univariate approaches to risk assessment for a large portfolio. It depends on the particular criterion which approach is better. It seems that, for one-day VaR and ES assessments, univariate modelling (of portfolio value instead of portfolio components) is often sufficient. For $s$ day VAR estimates (where $s=2,3, \ldots, 10$ ) the $n$-variate MSF-BEKK model turned out to be better (in terms of the Lopez loss function), but mean absolute errors for ES indicate that the univariate approaches are enough (especially for short trading positions).

Our empirical study shows that the simple hybrid SV-GARCH $(1,1)$ model, which is the univariate version of the MSF-BEKK $(1,1)$ model, behaves well and successfully competes with non-parametric specifications (CAViaR and CARE). Thus, our univariate hybrid model appears an interesting all-purpose alternative to different non-parametric models designed to focus on specific aspects of future returns (and not on their full predictive distribution).

Finally, the paper indicates that the Bayesian approach to VaR and ES analysis is fully relevant and practical. Remind that conditioning on observed data and inference on non-linear functions of unobserved quantities (future logarithmic returns) are necessary for any appropriate risk analysis. Both are natural and easy within Bayesian statistics, equipped with the Markov Chain Monte Carlo (MCMC) simulation tools.

\section{References}

[1] P. Artzner, F. Delbaen, J.-M. Eber, D. Heath, Mathematical Finance 9, 203 (1999).

[2] R. Engle, S. Manganelli, J. Bus. Econ. Stat. 22, 367 (2004).

[3] A. McNeil, R. Frey, J. Emp. Finance 7, 271 (2000).

[4] Y. Yamai, T. Yoshiba, Monetary Economic Studies 20, 95 (2002).

[5] J.W. Taylor, J. Financial Econometrics 6, 231 (2008). 
TABLE XI

$\operatorname{VaR}_{t: t+s}^{j}(0.05)$ and $\mathrm{ES}_{t: t+s}^{j}(0.05)$ results based on the univariate SV-GARCH model, $\omega_{\tau, i}=1 / 50$

\begin{tabular}{c|c|c|c|c|c|c|c|c|c|c|c}
\hline \hline & 1 & 2 & 3 & 4 & 5 & 6 & 7 & 8 & 9 & 10 \\
\hline \multicolumn{10}{|c|}{ s long trading position } \\
\hline FR & 0.04 & 0.03 & 0.04 & 0.04 & 0.045 & 0.04 & 0.035 & 0.005 & 0.01 & 0.005 \\
\hline$p$-value for the Kupiec test & 0.502 & 0.162 & 0.502 & 0.502 & 0.742 & 0.502 & 0.305 & 0.000 & 0.002 & 0.000 \\
\hline Lopez loss function & 146.97 & 385.70 & 356.18 & 147.67 & 52.09 & 133.13 & $\mathbf{2 5 . 0 3}$ & 6.83 & 3.20 & 2.19 \\
\hline ME & -13.777 & -35.838 & -12.598 & $\mathbf{1 9 . 4 5 2}$ & $\mathbf{4 5 . 2 7 5}$ & 40.261 & 77.449 & $\mathbf{4 7 . 2 9 8}$ & 82.062 & 89.645 \\
\hline MAE & $\mathbf{3 1 . 0 1 8}$ & 45.176 & $\mathbf{4 7 . 5 3 9}$ & 56.553 & $\mathbf{4 5 . 2 7 5}$ & $\mathbf{4 2 . 4 1 7}$ & 78.262 & $\mathbf{4 7 . 2 9 8}$ & 82.062 & 89.645 \\
\hline MAPE & $\mathbf{0 . 2 2 0}$ & 0.223 & $\mathbf{0 . 2 0 8}$ & 0.227 & $\mathbf{0 . 1 9 7}$ & 0.159 & 0.276 & $\mathbf{0 . 1 8 5}$ & 0.294 & 0.279 \\
\hline \multicolumn{8}{|c|}{ short trading position } \\
\hline FR & 0.045 & 0.04 & 0.04 & 0.035 & 0.04 & 0.03 & 0.04 & 0.055 & 0.045 & 0.035 \\
\hline$p$-value for the Kupiec test & 0.742 & 0.502 & 0.502 & 0.305 & 0.502 & 0.162 & 0.502 & 0.749 & 0.742 & 0.305 \\
\hline Lopez loss function & 46.04 & 130.36 & 234.13 & 401.39 & 437.66 & 349.11 & 160.75 & 153.94 & 238.99 & 199.22 \\
\hline ME & $\mathbf{1 0 . 1 2 4}$ & $\mathbf{1 4 . 3 8 5}$ & $\mathbf{9 . 5 6 1}$ & $\mathbf{- 0 . 2 9 2}$ & 22.372 & $\mathbf{1 5 . 9 6 6}$ & $\mathbf{5 8 . 1 0 7}$ & 88.012 & $\mathbf{7 3 . 8 6 6}$ & 87.931 \\
\hline MAE & $\mathbf{2 1 . 5 2 2}$ & 37.278 & $\mathbf{2 9 . 0 4 5}$ & $\mathbf{3 4 . 4 0 2}$ & $\mathbf{3 7 . 6 3 9}$ & $\mathbf{3 9 . 6 6 9}$ & $\mathbf{5 9 . 7 6 0}$ & 88.012 & $\mathbf{7 3 . 8 6 6}$ & 87.931 \\
\hline MAPE & $\mathbf{0 . 1 8 2}$ & 0.198 & $\mathbf{0 . 1 2 1}$ & $\mathbf{0 . 1 1 6}$ & $\mathbf{0 . 1 2 0}$ & $\mathbf{0 . 1 1 4}$ & $\mathbf{0 . 1 7 0}$ & 0.246 & $\mathbf{0 . 1 8 3}$ & 0.202
\end{tabular}

TABLE XII

$\operatorname{VaR}_{t: t+s}^{j}(0.05)$ and $\operatorname{ES}_{t: t+s}^{j}(0.05)$ results based on the $n$-variate MSF-SBEKK model, $\omega_{\tau, i}=1 / 50$

\begin{tabular}{c|c|c|c|c|c|c|c|c|c|c|c}
\hline \hline \multicolumn{1}{|c|}{} & 1 & 2 & 3 & 4 & 5 & 6 & 7 & 8 & 9 & 10 \\
\hline \multicolumn{8}{|c|}{ long trading position } \\
\hline FR & 0.055 & 0.04 & 0.035 & 0.025 & 0.015 & 0.01 & 0.015 & 0.005 & 0.000 & 0.000 \\
\hline$p$-value for the Kupiec test & 0.749 & 0.502 & 0.305 & 0.074 & 0.008 & 0.002 & 0.008 & 0.000 & - & - \\
\hline Lopez loss function & $\mathbf{1 0 6 . 3 4}$ & $\mathbf{3 2 9 . 2 0}$ & $\mathbf{2 5 8 . 9 6}$ & $\mathbf{7 0 . 4 2}$ & $\mathbf{4 . 8 7}$ & $\mathbf{1 0 8 . 3 7}$ & 42.37 & $\mathbf{1 . 5 4}$ & $\mathbf{0 . 0 0}$ & $\mathbf{0 . 0 0}$ \\
\hline ME & $\mathbf{3 . 7 8 1}$ & $\mathbf{- 1 6 . 2 1 0}$ & $\mathbf{7 . 5 1 2}$ & 39.301 & 70.779 & $\mathbf{1 . 1 4 7}$ & $\mathbf{4 7 . 5 1 0}$ & 99.901 & - & - \\
\hline MAE & 37.377 & $\mathbf{3 6 . 0 6 7}$ & 54.457 & $\mathbf{4 8 . 0 3 8}$ & 70.779 & 48.583 & $\mathbf{6 5 . 2 2 7}$ & 99.901 & - & - \\
\hline MAPE & 0.305 & $\mathbf{0 . 1 9 8}$ & 0.237 & $\mathbf{0 . 1 7 8}$ & 0.255 & $\mathbf{0 . 1 4 1}$ & $\mathbf{0 . 1 8 6}$ & 0.283 & - & - \\
\hline \multicolumn{7}{|c|}{ short trading position } \\
\hline FR & 0.05 & 0.045 & 0.04 & 0.03 & 0.025 & 0.03 & 0.03 & 0.015 & 0.025 & 0.01 \\
\hline -value for the Kupiec test & 1.000 & 0.742 & 0.502 & 0.162 & 0.074 & 0.162 & 0.162 & 0.008 & 0.074 & 0.002 \\
\hline Lopez loss function & $\mathbf{3 0 . 7 3}$ & $\mathbf{5 9 . 9 7}$ & $\mathbf{1 3 6 . 3 5}$ & $\mathbf{2 2 6 . 1 6}$ & $\mathbf{2 8 9 . 1 0}$ & $\mathbf{1 9 2 . 8 6}$ & $\mathbf{1 2 . 4 7}$ & $\mathbf{2 8 . 4 8}$ & $\mathbf{3 3 . 8 0}$ & $\mathbf{4 3 . 4 1}$ \\
\hline ME & 16.209 & 21.160 & 21.259 & 20.884 & $\mathbf{8 . 2 6 9}$ & 55.373 & 89.714 & $\mathbf{6 8 . 9 1 0}$ & 92.682 & $\mathbf{5 3 . 4 3 6}$ \\
\hline MAE & 27.287 & $\mathbf{3 3 . 0 8 4}$ & 35.493 & 45.690 & 45.162 & 55.968 & 89.714 & $\mathbf{6 8 . 9 1 0}$ & 92.682 & $\mathbf{5 3 . 4 3 6}$ \\
\hline MAPE & 0.271 & $\mathbf{0 . 1 9 2}$ & 0.146 & 0.145 & 0.133 & 0.158 & 0.264 & $\mathbf{0 . 1 7 3}$ & 0.222 & $\mathbf{0 . 1 2 2}$
\end{tabular}

[6] L. Bauwens, S. Laurent, J.V.K. Rombouts, J. Appl. Econometrics 21, 79 (2006).

[7] R.S. Tsay, Analysis of Financial Time Series $\left(2^{\text {nd }}\right.$ edition), Wiley, New York 2005.

[8] J. Osiewalski, Przeglad Statystyczny 56, 15 (2009).

[9] J. Osiewalski, A. Pajor, Central European J. Economic Modelling Econometrics 1 (2), 179 (2009).

[10] A. Pajor, Dynamic Econometric Models 9, 81 (2009).

[11] J. Osiewalski, A. Pajor, Central European Journal of Economic Modelling and Econometrics 2 (4), 253 (2010).

[12] A. O'Hagan, Bayesian Inference, Edward Arnold, London 1994.

[13] A. Pajor, Acta Universitatis Lodziensis - Folia Oeconomica 190, 177 (2005).
[14] J.A. Lopez, Federal Reserve Bank of San Francisco Economic Review 2, 3 (1999).

[15] M. Sarma, S. Thomas, A. Shah, J. Forecasting 22 (4), 337 (2003).

[16] T.H. Lee, in: International Encyclopedia of the Social Sciences, 2nd ed., vol. 4, , Ed. W.A. Darity, Macmillan Thomson Gale, Detroit 2008, p. 495.

[17] D. Zhu, J.W. Galbraith, www.cirano.qc.ca/pdf/ publication/2009s-24.pdf .

[18] R. Kaufmann, P. Patie, Strategic Long-Term Financial Risks - The One-Dimensional Cases, RiskLab Report, ETH Zurich, (2003). Available at http: //www.risklab.ch/Papers.html\\#SLTFR.

[19] P. Embrechts, R. Kaufmann, P. Patie, Computational Optimization and Applications 32 (1/2), 61 (2005). 